\title{
Does Video Laryngoscopy or Direct Laryngoscopy affect first pass success rates for intubation among Attending and Non-Attending Emergency Physician in the Emergency Department?
}

Paul Weng Wan ( $\nabla$ wan.paul.weng@singhealth.com.sg )

Singhealth https://orcid.org/0000-0002-0030-1140

Zakaria Nur Diana

Singhealth

Seow Gek Ching

Singhealth

Wong Evelyn

Singhealth

Research article

Keywords: Laryngoscopy, intubation, first pass success, experience, difficult intubation

Posted Date: August 23rd, 2019

DOl: https://doi.org/10.21203/rs.2.13427/v1

License: (c) (1) This work is licensed under a Creative Commons Attribution 4.0 International License.

Read Full License 


\section{Abstract}

Background: To our knowledge, there has been no study comparing intubation characteristics between attending and non-attending Emergency Physicians in South-East Asia. We aim to identify whether the use of Direct Laryngoscopy (DL) compared to Video Laryngoscopy (VL) affects first pass success rates between Attending Emergency Physicians (AEP) and Non-Attending Emergency Physicians (NAEP).

Materials and Methods: Retrospective analysis of data from 2009 to 2016 in an existing airway registry managed by and academic Emergency Department in Singapore. Primary outcome is first pass success intubation rate. The secondary outcome was first pass success rate for difficult intubations. Difficult intubations were defined as LEMON score of more than 1 or more than 1 attempt at intubation.

Results: There were 2909 intubation carried out by emergency physicians in the Emergency Department from 2009 to 2016. AEP conducted 1748 intubations while NAEP conducted 1161 intubations. The first pass success rates for AEP was $84.2 \%$ while that for NAEP was $67.4 \% .86 .2 \%$ of intubations by AEP were done with a direct laryngoscope. $89.0 \%$ of the intubations by NAEP were done with a direct laryngoscope. $18.9 \%$ of intubations by the AEP were difficult compared to $35.2 \%$ by the NAEP ( $<<0.0195 \%$ C.I $13.0 \%-19.6 \%$ ). First pass success rate with VL was lower than DL for all intubations (OR $0.66,95 \%$ C.I 0.51-0.84). In the subgroup of difficult intubations, VL did not improve first pass success rate among AEP (OR 0.77, 95\% C.I 0.38-1.58) but it did for NAEP (OR 2.46, 95\% C.I 0.94-6.45).

Conclusion: Our study showed that VL has a poorer first pass success rate for all intubations in general. However, specifically for difficult intubations, VL is associated with improved first pass success rates among NAEP.

\section{Introduction}

First pass success in emergency intubations has been associated with fewer adverse events ${ }^{1}$ than if more than a single attempt is needed at intubation. Level of experience and choice of Video Laryngoscopy $(\mathrm{VL})$ or Direct Laryngoscopy $(\mathrm{DL})$ have been reported to be features associated with first pass success in intubations ${ }^{2,3,4}$. In South-East Asia, there have been no studies examining the relationship between these two factors and how they relate to first pass success in intubation. We aim to identify whether the use of VL compared to $D L$ affects first pass success rates for regular or difficult endotracheal intubations when performed by Attending Emergency Physicians (AEP) and Non-Attending Emergency Physicians (NAEP).

\section{Materials And Methods}


This was a single-center retrospective observational study. Data was obtained from the Airway Registry of an academic Emergency Department (ED) at the Singapore General Hospital (SGH), Singapore, between 2009 and 2016. All intubations conducted in the ED during this period were documented on a standardized hardcopy form. The entries were then transcribed into an electronic Airway Registry. Approval from the Institution Review Board was obtained for data collection into the Airway Registry.

For this study, only intubations done by Emergency Physicians were analysed. Intubations performed in the ED by other operators, e.g. Anaesthetists, were excluded.

Attending Emergency Physicians (AEP) are senior physicians who have completed Residency training and are board certified in Emergency Medicine by the Specialist Accreditation Board of the Ministry of Health, Singapore. Non-attending Emergency Physicians (NAEP) are physicians who are not - these include Emergency Medicine Residents, and Medical Officers who are not in a Residency Programme. All intubations by NAEP were supervised by AEP.

The VLs used during this period were the GlideScope ${ }^{\circledR}$ (Verathon Medical Inc., Bothell, USA), C-MAC (Karl Storz GmbH \& Co. KG, Tuttlingen, Germany) and McGrath (Aircraft Medical, UK). DL was carried out with the Macintosh Curved or Miller Straight Blade (Welch Allyn, NY).

The primary outcome was rate of successful first pass intubation. A single pass was defined as an attempt to pass the endotracheal tube (ETT) through the vocal cords. A pass will usually be followed by airway maneuvers e.g. bagging or suctioning.

The LEMON (Look externally, Evaluate 3-3-2, Mallampati, Obstruction, Neck Mobility) criteria is a commonly used tool to assess a difficult airway. The presence of any of these features in the criteria will be given a score of 1 . A difficult intubation is defined as a LEMON score of more than 1 or the number of attempts required for a successful intubation being more than 1 .

Secondary outcomes were the first pass intubation success rate for difficult intubations.

Statistical calculations were performed using SPSS 26.0 for Windows (IBM Corp. Released 2019. IBM SPSS Statistics for Windows, Version 26.0. Armonk, NY: IBM Corp). Continuous and ordinal variables were presented as means and categorical variables as frequencies. Categorical variables were analysed 
using Chi-Squared Test for differences between groups. The t-test was used to identify differences between groups for continuous variables. Statistical significance was considered if $p<0.05$. Odds Ratios (OR) and 95\% confidence intervals $(95 \% \mathrm{Cl})$ were presented where applicable.

\section{Results}

\section{Patient Characteristics}

There were 2950 intubations carried out at the ED, SGH from 2009 to 2016. Of these, 2909 (98.6\%) were conducted by Emergency Physicians with 1748 (59.3\%) intubations by AEPs and 1161 (39.3\%) by NAEPs (Table 1). Those patients intubated by NAEPs were slightly older than those intubated by AEPs (63.5 versus 60.7 years, $p<0.01)$. Gender distribution between the groups was similar. AEPs performed more trauma intubations than NAEPs (13.6\% versus $8.5 \%)$. NAEPs encountered more difficult intubations than did AEPs (35.2\% versus $18.9 \%)$.

\section{All Intubations}

AEPs had a higher overall first pass success rate than NAEPs $(84.2 \%$ versus $67.4 \%, p<0.01)$. The mean number of attempts was 1.24 and 1.46 for AEPs and NAEPs, respectively.

The first pass success rate for all intubations was lower for VL (70.6\%) than for DL $(78.5 \%)(p=0.0008$, OR $0.66,95 \% \mathrm{Cl} 0.51-0.84)$. However, for the subgroup of difficult intubations, there was a slightly higher, though not statistically significant, success rate for VL $(13.8 \%)$ versus $D L(10.6 \%)$ for all practitioners $(p=$ 0.3081 , OR $1.34,95 \% \mathrm{Cl} 0.76-2.39)$.

AEPs were also more likely to use video laryngoscopy than NAEPs $(p=0.0139)$. Among AEPs, VL had a lower first pass success rate when compared to use of $D L(70.6 \%$ versus $86.3 \%, p=0.0001,0 R 0.38$, $95 \% \mathrm{Cl} 0.28$ - 0.52). Among NAEP (Table 2), there was no difference in first pass success rates whether VL $(70.5 \%)$ or DL $(67.1 \%)$ was used $(p=0.4481$, OR $1.17,95 \% \mathrm{Cl} 0.78-1.77)$.

\section{Difficult Intubations}


Altogether, there were 739 difficult intubations. Of these 330 were encountered by AEPs and 409 by NAEPS.

For all patients with a LEMON score of $\leq 1$, first pass success rate was $85.6 \%$ by AEPs and $69.0 \%$ by NAEPs $(p<0.0001$, OR $2.68,95 \% C l 2.22-3.23)$.

Patients with a LEMON score of $>1$ were encountered by $5.3 \%$ of AEPs and $6.1 \%$ of NAEPs $(p=0.32)$. For all patients with a LEMON score $>1$, the first pass success rate was $58.7 \%$ when intubated by AEPs and $42.2 \%$ when intubated by NAEPs ( $p=0.04$, OR $1.94,95 \% \mathrm{Cl} 1.04-3.64)$.

However, with difficult intubations, though VL appeared to have a higher first pass success rate (13.8\%) compared to DL (10.6\%), this difference did not reach statistical significance $(\mathrm{p}=0.3081, \mathrm{OR} 1.34,95 \% \mathrm{Cl}$ 0.76-2.39). First pass success rates did not differ for VL-assisted difficult intubations by AEPs and NAEPs (13.6\% vs $14.2 \%, p=0.9144$, OR $0.94,95 \% \mathrm{Cl} 0.32-2.76)$. For difficult intubations done with $\mathrm{DL}$, AEPs had a higher first pass success rate (16.9\%) than NAEPs (6.3\%) ( $p=0.0001$, OR 3.01, 95\% Cl 1.76 - 5.16).

\section{Video Laryngoscopy}

The GlideScope had the highest first pass success rate among all VL (OR 2.85, 95\% C.I 0.39-20.7), followed by C-MAC (OR 1.79 95\% C.I 0.24-13.14) and lastly McGrath. For each variant of VL used, there was no statistically significant difference in the first past success rates between AEP and NAEP (Table 3). This finding was also consistent regardless of whether it was a difficult intubation.

\section{Discussion}

The observed finding of VL having lower overall first pass success rate compared to $D L(p=0.0008$, OR $0.6695 \%$ C.I $0.51-0.84$ ) is dissimilar to existing literature. Existing publications 6,7,8,9,10 report no statistically significant difference in first pass success rates between VL and DL. The greater familiarity with DL likely accounted for the higher rates of DL use in our study and the lower rates of first pass success with VL.

For difficult intubations, VL was associated with marginally better overall first pass success rates $(p=$ 0.3081, OR 1.34, 95\% C.I 0.76-2.39). With VL, the GlideScope had the highest first pass success rate compared to the other two VL. The presence of a video camera at the distal end of the VL allows for 
visualization of the vocal cords without alignment of the various intubation axes. Specifically, the rigid stylet for the GlideScope Video Laryngoscope allows for smooth insertion ${ }^{11,12}$ of the ETT along the hyperacute angle of the laryngoscope blade. These features likely helped improve first pass success rates in difficult intubations $7,11,12,13$, especially where small mouth opening, and limited neck mobility posed a challenge. The C-Mac allows the user to perform intubation as per DL or via its attached video camera with an external monitor. The McGrath has a monitor attached to the laryngoscopy blade, allowing the user to maintain line of sight of the patient and hand positions while intubating. Despite the various traits of each device, our study did not show statistically significant improved first pass intubation rates when these devices were used by AEPs than by supervised NAEPs (Table 3).

Moreover, these results are further augmented in the subgroup of difficult intubations among the NAEP $7,11,12,13$. An improved glottic view and ease in anatomy identification are reported mechanisms resulting in success with VL among NAEP for difficult intubations. In addition, the ability to visualize the NAEP's field of view with VL also allows the supervising AEP to provide timely and appropriate advice during the intubation. Therefore, video laryngoscope-assisted intubations are useful for training relative novices in endotracheal intubations skills, especially during difficult intubations by these groups of healthcare workers. ${ }^{14,15}$

Our study clearly demonstrated that persons performing endotracheal intubations performed better with devices they were familiar with, such as with direct laryngoscopy. Therefore, trained AEPs did better, as would be expected, than NAEPs with DL and also used DL more effectively than VL. However, when encountering difficult airways the large differences in first-pass successful intubation rates between DL and VL were greatly reduced. These attest to the need for training with use of VL and to the use of VL as a secondary adjunct for gaining airway access during difficult intubations.

First pass success during Emergency Intubations are associated with fewer adverse effects and better patient outcomes ${ }^{14,15}$. Therein lies the importance to balance operator level of training and device choice so that an optimal first pass success rate is achieved. If novice operators are not provided with the opportunity to conduct Emergency Intubations, this may eventually affect their first pass success rates as future airway managers. 


\section{Limitations}

This was a single-center study with data derived from an Airway Registry. Cases were entered into the Airway Registry by manually transcribing into the electronic database from a hard copy form. This allows for errors in transcription. In addition, since the forms were completed by the operators after completion of the resuscitation which included the intubation, recall bias may contribute to inaccurately recorded data. This may thus affect the generalizability of the results. Of note, our results specifically for the difficult intubation subgroup are consistent with that of existing literature.

Our definition of a difficult intubation did not use a single standardized definition such as the Intubation

Difficulty Score (IDS) ${ }^{16}$. In addition to the LEMON score, we included all first pass intubation failures. The greater unfamiliarity of NAEPs with endotracheal intubation likely contributed to the higher proportion of difficult intubations in this group and the larger overall number of such intubations. The LEMON score, by itself, has been shown to correlate well with difficulty of intubation ${ }^{17,18}$.

\section{Conclusions}

Our study shows that VL has a poorer first pass success rate for all intubations in general. However, specifically for difficult intubations, VL is associated with improved first pass success rates especially when used by supervised NAEPs. Between AEPs and NAEPs, while DL use was easier with trained AEPs, whether for standard or difficult airways, there was no statistically significant difference in first pass intubation rates with VL use.

\section{Declarations}

Ethics Approval and Consent to Participate

Approval from the Singhealth Institution Review Board was obtained for data collection into the Airway Registry used for this study.

Consent for Publication 
Not Applicable

Availability of Data and Materials

The dataset analysed in this current study may be made available on reasonable request. Please contact WPW.

Competing Interests

There are no competing interests to declare.

\section{Funding}

Not applicable

Authors' Contributions

WPW, NDZ, EW developed the study and supervised its data collection. SGC assisted with data collection and entry. WPW analysed the data. WPW drafted the manuscript. NDZ and EW provided advice the manuscript and contributed as corresponding authors. WPW take responsibility for the paper.

Acknowledgements

The authors would like to thank Prof. V. Anantharaman for his guidance and support in writing this manuscript.

\section{References}

1. Sakles J, Chiu S, Mosier J, Walker C, Stolz U. The Importance of First Pass Success When Performing Orotracheal Intubation in the Emergency Department. Academic Emergency Medicine. 2013;20(1):7178.

2. Okamoto H, Goto T, Wong Z, Hagiwara $Y$, Watase H, Hasegawa K. Comparison of video laryngoscopy versus direct laryngoscopy for intubation in emergency department patients with cardiac arrest: $\mathrm{A}$ multicentre study. Resuscitation. 2019;136:70-77. 
3. Sakles J, Mosier J, Chiu S, Keim S. Tracheal Intubation in the Emergency Department: A Comparison of GlideScope ${ }^{\circledR}$ Video Laryngoscopy to Direct Laryngoscopy in 822 Intubations. The Journal of Emergency Medicine. 2012;42(4):400-405.

4. Vassiliadis J, Tzannes A, Hitos K, Brimble J, Fogg T. Comparison of the C-MAC video laryngoscope with direct Macintosh laryngoscopy in the emergency department. Emergency Medicine Australasia. 2015;27(2):119-125.

5. Bhattacharjee S, Maitra S, Baidya D. A comparison between video laryngoscopy and direct laryngoscopy for endotracheal intubation in the emergency department: A meta-analysis of randomized controlled trials. Journal of Clinical Anesthesia. 2018;47:21-26.

6. Choi H, Kim Y, Oh Y, Kang H, Yim H, Jeong S. GlideScope video laryngoscopy versus direct laryngoscopy in the emergency department: a propensity score-matched analysis. BMJ Open. 2015;5(5):e007884-e007884.

7. Choi H, Kang H, Lim T, Chung H, Cho J, Oh Y et al. Endotracheal intubation using a GlideScope video laryngoscope by emergency physicians: a multicentre analysis of 345 attempts in adult patients. Emergency Medicine Journal. 2010;27(5):380-382.

8. Lewis S, Butler A, Parker J, Cook T, Smith A. Videolaryngoscopy versus direct laryngoscopy for adult patients requiring tracheal intubation. Cochrane Database of Systematic Reviews. 2016;

9. Driver B, Prekker M, Moore J, Schick A, Reardon R, Miner J. Direct Versus Video Laryngoscopy Using the C-MAC for Tracheal Intubation in the Emergency Department, a Randomized Controlled Trial. Academic Emergency Medicine. 2016;23(4):433-439.

10. Lee Y, Chen C, Wang T, Lin K, Su Y. Comparison of video and direct laryngoscope for tracheal intubation in emergency settings: A meta-analysis. Journal of Acute Medicine. 2012;2(2):43-49.

11. Yeatts $D$, Dutton $R$, Hu P, Chang $Y$, Brown $C$, Chen $H$ et al. Effect of video laryngoscopy on trauma patient survival. Journal of Trauma and Acute Care Surgery. 2013;75(2):212-219.

12. Michailidou M, O'Keeffe T, Mosier J, Friese R, Joseph B, Rhee P et al. A Comparison of Video Laryngoscopy to Direct Laryngoscopy for the Emergency Intubation of Trauma Patients. World Journal of Surgery. 2014;39(3):782-788.

13. Mosier J, Stolz U, Chiu S, Sakles J. Difficult Airway Management in the Emergency Department: GlideScope Videolaryngoscopy Compared to Direct Laryngoscopy. The Journal of Emergency Medicine. 2012;42(6):629-634.

14. Howard-Quijano K, Huang Y, Matevosian R, Kaplan M, Steadman R. Video-assisted instruction improves the success rate for tracheal intubation by novices. British Journal of Anaesthesia. 2008;101(4):568-572.

15. Sakles J, Javedani P, Chase E, Garst-Orozco J, Guillen-Rodriguez J, Stolz U. The Use of a Video Laryngoscope by Emergency Medicine Residents Is Associated With a Reduction in Esophageal Intubations in the Emergency Department. Academic Emergency Medicine. 2015;22(6):700-707.

16. Adnet F, Borron S, Racine S, Clemessy J, Fournier J, Plaisance P et al. The Intubation Difficulty Scale (IDS). Anesthesiology. 1997;87(6):1290-1297. 
17. Ji S, Moon E, Kim T, Yi J, Seo H, Lee B. Correlation between modified LEMON score and intubation difficulty in adult trauma patients undergoing emergency surgery. World Journal of Emergency Surgery. 2018;13(1).

18. Ogboli-Nwasor E, Mshelia D, Isamade E. Use of the "L-E-M-O-N" score in predicting difficult intubation in Africans. Nigerian Journal of Basic and Clinical Sciences. 2018;15(1):17.

19. Cho Y, Cho J, Chung H. Assessment of Emergency Airway Management Techniques in Korea Using an Online Registration System: A Multicenter Study. The Journal of Emergency Medicine. 2015;48(1):1-9.

\section{Tables}

Table 1: Baseline Characteristics 


\begin{tabular}{|c|c|c|c|}
\hline & $\begin{array}{l}\text { Attending } \\
(1748)\end{array}$ & $\begin{array}{c}\text { Non-attending } \\
(1161)\end{array}$ & p Value \\
\hline $\begin{array}{l}\text { Gender } \\
\text { Female } \\
\text { Male }\end{array}$ & $\begin{array}{c}560(32.0 \%) \\
1188(68.0 \%)\end{array}$ & $\begin{array}{l}384(33.1 \%) \\
777(66.9 \%)\end{array}$ & 0.56 \\
\hline Mean age (years) & $60.7(0.1-100.0)$ & $63.5(7.0-110.0)$ & $<0.01$ \\
\hline $\begin{array}{l}\text { Medical/trauma* } \\
\text { Medical } \\
\text { Trauma }\end{array}$ & $\begin{array}{c}1509(86.4 \%) \\
237(13.6 \%)\end{array}$ & $\begin{array}{c}1062(91.5 \%) \\
99(8.5 \%)\end{array}$ & $<0.01$ \\
\hline $\begin{array}{l}\text { Device used } \\
\text { Direct laryngoscope }\end{array}$ & $\begin{array}{c}1506 \\
1486(98.7 \%) \\
20(1.3 \%)\end{array}$ & $\begin{array}{c}1033 \\
1028(99.5 \%) \\
5(0.5 \%)\end{array}$ & 0.01 \\
\hline Video laryngoscope & $\begin{array}{c}238 \\
162(68.1 \%) \\
4(1.7 \%) \\
72(30.2 \%)\end{array}$ & $\begin{array}{c}122 \\
77(63.1 \%) \\
0(0.0 \%) \\
45(36.9 \%)\end{array}$ & \\
\hline Others** & $4(0.2 \%)$ & $6(0.5 \%)$ & \\
\hline Crash intubation & $804(46.0 \%)$ & $493(42.5 \%)$ & 0.06 \\
\hline RSI with medications & $944(54.0 \%)$ & $668(57.5 \%)$ & 0.06 \\
\hline 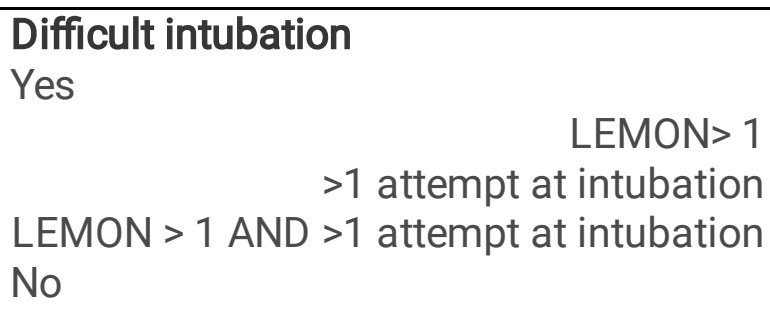 & $\begin{array}{c}330(18.9 \%) \\
54(16.4 \%) \\
238(72.1 \%) \\
38(11.5 \%) \\
1418(81.1 \%)\end{array}$ & $\begin{array}{c}409(35.2 \%) \\
30(7.3 \%) \\
338(82.6 \%) \\
41(10.0 \%) \\
752(64.8 \%)\end{array}$ & $<0.01$ \\
\hline First pass success rate (\%) & $84.2 \%(1472 / 1748)$ & $67.4 \%(782 / 1161)$ & $<0.01$ \\
\hline Mean number of attempts & $1.24(1.21-1.27)$ & 1.46(1.41-1.50) & $<0.01$ \\
\hline
\end{tabular}

*2 cases were not included as the data was unavailable ** Data was not available on the specific device used, not included in analysis in VL, DL groups. 
Table 2: First Pass Success Rates

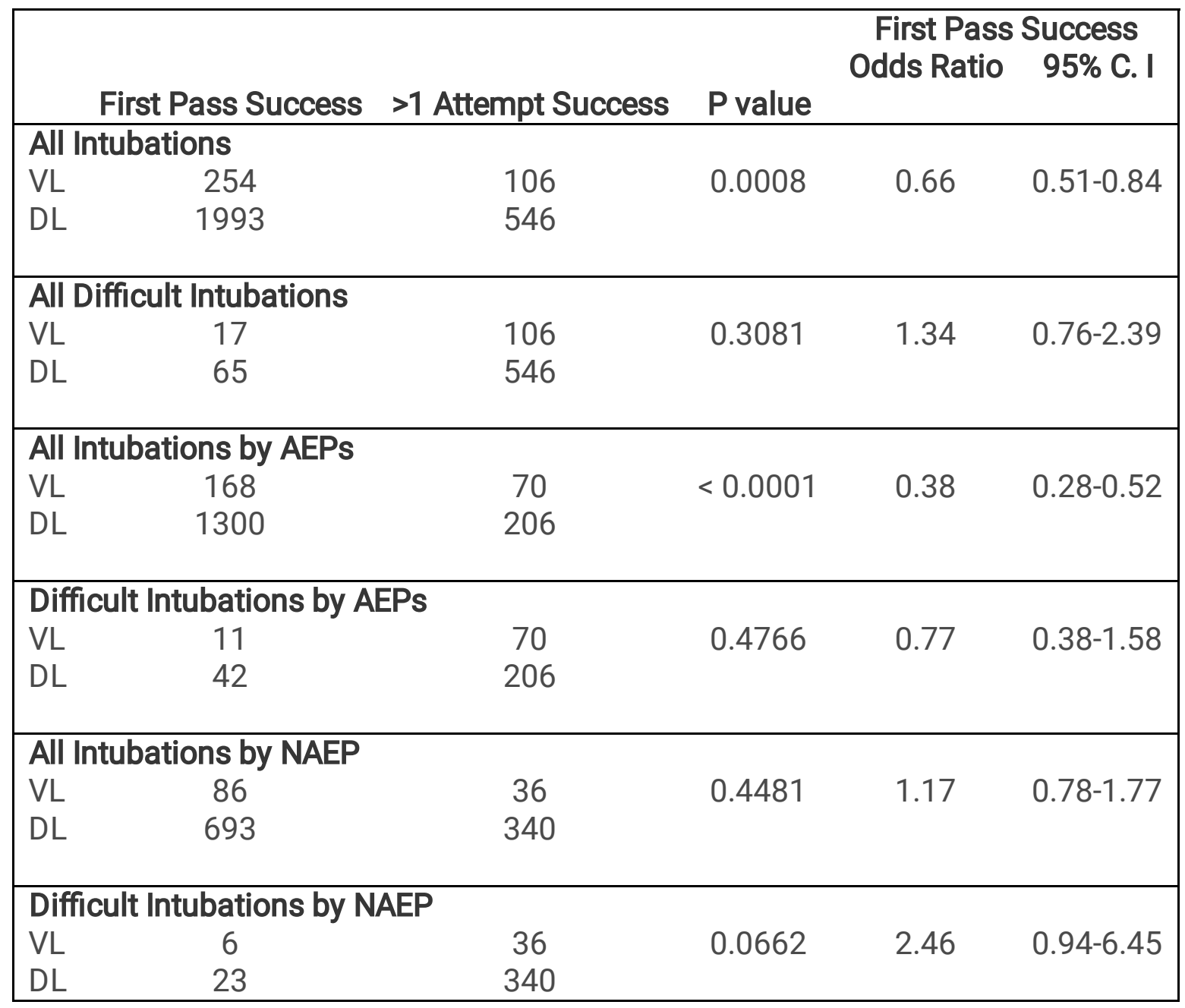


Table 3: First Pass Success Rate for various VL

\begin{tabular}{|c|c|c|c|c|}
\hline & & & First Pas & Success \\
\hline & First Pass Success & $>1$ Attempt Success & Odds Ratio & 95\% C. I \\
\hline All VL & & & & \\
\hline McGrath & 2 & 2 & Reference & \\
\hline C-MAC & 75 & 42 & 1.79 & $0.24-13.14$ \\
\hline GlideScope & 177 & 62 & 2.85 & $0.39-20.7$ \\
\hline GlideScope & & & & \\
\hline AEP & 119 & 43 & 0.91 & $0.49-1.69$ \\
\hline NAEP & 58 & 19 & & \\
\hline GlideScope & and Difficult Intubatio & & & \\
\hline AEP & 9 & 43 & 1.33 & $0.32-5.45$ \\
\hline NAEP & 3 & 19 & & \\
\hline C-MAC & & & & \\
\hline AEP & 47 & 25 & 1.14 & $0.53-2.47$ \\
\hline NAEP & 28 & 17 & & \\
\hline C-MAC and & ifficult Intubation & & & \\
\hline AEP & 2 & 25 & 0.45 & 0.07-3.01 \\
\hline NAEP & 3 & 17 & & \\
\hline
\end{tabular}

